apgs@ufv.br

Universidade Federal de Viçosa

Brasil

\title{
Governança aplicada às tecnologias sociais para populações em situação de risco socioeconômico associada ao paradigma da sustentabilidade
}

Castilhos, Alexandre; Silva, Tania Nunes da

Governança aplicada às tecnologias sociais para populações em situação de risco socioeconômico associada ao paradigma da sustentabilidade

Administração Pública e Gestão Social, vol. 12, núm. 2, 2020

Universidade Federal de Viçosa, Brasil

Disponible en: http://www.redalyc.org/articulo.oa?id=351562414008

Esta obra está bajo una Licencia Creative Commons Atribución-NoComercial-SinDerivar 3.0 Internacional. 


\title{
Governança aplicada às tecnologias sociais para populações em situação de risco socioeconômico associada ao paradigma da sustentabilidade
}

\author{
Governance applied to social technologies for populations at socioeconomic risk associated with the sustainability \\ paradigm \\ Gobernanza aplicada a las tecnologías sociales para las poblaciones de riesgo socioeconómico asociada con el \\ paradigma de la sostenibilidad
}

Alexandre Castilhos

Universidade Federal do Rio Grande do Sul, Brasil

Redalyc: http://www.redalyc.org/articulo.oa?

alexandre_castilhos@outlook.com

Tania Nunes da Silva

PPGA/Universidade Federal do Rio Grande do Sul, Brasil

tnsilva@ea.ufrgs.br id $=351562414008$

Recepción: 05 Junio 2017

Aprobación: 15 Marzo 2019

Publicación: 01 Abril 2020

\section{Resumo:}

O objetivo deste artigo é apresentar os resultados de uma pesquisa estruturada a partir de uma perspectiva qualitativa, a fim de identificar os parâmetros iniciais para o desenvolvimento de uma abordagem de governança das tecnologias sociais associada à sustentabilidade, considerando a perspectiva multinível. A revisão de literatura compreendeu os eixos temáticos relacionados à governança, tecnologias sociais e abordagem multinível. A abordagem de governança buscou relacionar informações obtidas junto às Instituições de Tecnologias Sociais no Brasil e, em um segundo momento, com as verificadas junto a outros dois grupos, as instituições formuladoras de políticas públicas em tecnologias sociais no Brasil e as instituições que adotam governança em tecnologias sociais no Brasil. Os resultados apontam para uma abordagem que busca uma nova forma de olhar a dinâmica das tecnologias sociais, problemas, inovações, modelos, estágios, transições, desafios e mudanças, a fim de contribuir para novos modelos de desenvolvimento social sustentável no Brasil.

Palavras-Chave: Sustentabilidade, Governança, Tecnologia social, Desenvolvimento, Políticas públicas.

\section{RESUMEN:}

El objetivo de este artículo consiste en presentar los resultados de una investigación estructurada desde una perspectiva cualitativa, a fin de identificar los parámetros iniciales para el desarrollo de un enfoque de gobernanza de las tecnologías sociales asociada a la sostenibilidad, considerando la perspectiva multi-nivel. La revisión de literatura comprendió los ejes temáticos relacionados con la gobernanza, las tecnologías sociales y el enfoque multi-nivel. El enfoque de gobernanza buscó relacionar informaciones obtenidas junto a las Instituciones de Tecnologías Sociales en Brasil y, en un segundo momento, con las verificadas junto a otros dos grupos: i) Las instituciones formuladoras de políticas públicas en tecnologías sociales en Brasil y ii) las instituciones que adoptan la gobernanza en tecnologías sociales en Brasil. Los resultados apuntan a un enfoque que busca una nueva forma de mirar la dinámica de las tecnologías sociales, problemas, innovaciones, modelos, etapas, transiciones, desafíos y cambios, a fin de contribuir para la elaboración de nuevos modelos de desarrollo social sostenible en Brasil.

Palabras Clave: Sostenibilidad, Gobernanza, Tecnología social, Desarrollo, Políticas públicas.

\section{Abstract:}

The purpose of this paper is to present the results of a research, structured from a qualitative perspective, in order to identify the initial parameters for the development of a governance approach in social technologies associated with sustainability, considering the multilevel perspective. The theoretical framework included the topics related to governance, social technologies and multilevel approach. The governance approach sought to relate data obtained from the Social Technology Institutions in Brazil and from two other groups: i) The institutions of public policies formulation in social technologies in Brazil and ii) the institutions that adopt governance in social technologies in Brazil. The results showed an approach that seeks a new way of analysing the dynamics 
of social technologies, problems, innovations, models, stages, transitions, challenges and changes, in order to contribute to new models of sustainable social development in Brazil.

KEYWORDS: Sustainability, Governance, Social technology, Development, Public policy.

\section{INTRODUÇÃo}

O avanço tecnológico é, atualmente, um dos principais fatores responsáveis pelo desenvolvimento econômico da sociedade moderna. Entretanto, este modelo de desenvolvimento gera impactos para a sustentabilidade do planeta, ocasionando desequilíbrios socioeconômicos, aumento da desigualdade social e impactos ambientais que podem causar graves prejuízos às gerações futuras.

Os impactos nos âmbitos social, econômico, tecnológico e ambiental gerados pelo avanço tecnológico tradicional são críticos e merecem atenção sob um olhar que considere novos modelos tecnológicos de base social associados à inclusão, governança e sustentabilidade. $\mathrm{O}$ campo de pesquisa sobre estes fenômenos, tratados de maneira integrada, ainda é restrito. Assim, é necessário entender melhor como lidar com os impactos gerados pela adoção de novas tecnologias no âmbito do avanço tecnológico tradicional, principalmente sob o ponto de vista da exclusão social, a fim de reverter esse efeito e possibilitar alternativas de inserção às populações em vulnerabilidade socioeconômica na economia.

Como forma de analisar novas abordagens que pudessem atender à dinâmica de desenvolvimento e sustentabilidade no contexto apresentado, este estudo explorou, inicialmente, a construção da abordagem de governança voltada às tecnologias sociais em conjunto com a perspectiva multinível de Geels e Kemp (2007) para populações em risco socioeconômico, a ser utilizada inicialmente para atender às demandas da realidade brasileira.

A partir deste enfoque, o estudo buscou tratar sobre os conceitos de governança e tecnologias sociais, no sentido de apresentar referências teóricas atuais e relevantes para sustentar as premissas da investigação realizada. Esta nova abordagem de governança tem como objetivo promover ações voltadas à inserção de comunidades vulneráveis na economia através do incentivo ao desenvolvimento e da implementação e expansão de tecnologias sociais dentro do paradigma da sustentabilidade.

Para que tais fatores se desenvolvam de maneira sistêmica, sustentável, eficiente e eficaz, é necessária uma nova governança, comprometida, séria, ética e estruturada para assegurar a realização do processo de inclusão. Neste sentido, uma nova combinação de disciplinas se faz necessária para a composição de um modelo de governança aplicada às tecnologias sociais. Esta combinação reúne abordagens inter, multi e transdisciplinares para obter respostas válidas diante do nível de complexidade proveniente dos atores, dinâmicas e ambientes envolvidos. A contribuição para as áreas de desenvolvimento social, gestão e sustentabilidade, a partir desta nova organização, é de fundamental importância, pois contempla uma nova perspectiva de inclusão para os atores sociais, minimizando riscos, incentivando inovação social através de novas formas de tecnologias e promovendo accountability (ética e transparência na prestação de contas).

Logo, o presente estudo identificou a necessidade de aprofundar a compreensão dos temas referentes aos desenvolvimentos social, tecnológico e econômico à inclusão social, bem como à sustentabilidade, que estão inter-relacionados com a governança em tecnologias sociais. Esta necessidade suscitou a seguinte questão de pesquisa: Como a governança em tecnologias sociais pode ser associada à sustentabilidade, a fim de constituir uma nova abordagem que possa servir de referência e também contribuir para reverter o quadro de populações em situação de risco socioeconômico?

Este artigo é composto por sete seções. Nesta primeira seção foi apresentada uma introdução ao tema abordado. $\mathrm{Na}$ segunda seção são apresentados os fundamentos teóricos, que compõem uma revisão de literatura que estabelece a base para o desenvolvimento da abordagem relacionada às tecnologias sociais, o que compreende os seguintes eixos: i) Governança; ii) Tecnologias Sociais, considerando a literatura sobre o conceito adotado no Brasil; e iii) Abordagem Multinível de Geels e Kemp (2007), que apresenta 
a interdependência dos elementos para a formação da abordagem e o entendimento das dinâmicas entre eles. $\mathrm{Na}$ terceira seção são apresentados os procedimentos metodológicos empregados. Na quarta seção são apresentados os resultados obtidos. Na quinta seção é apresentada uma discussão dos elementos teóricos e empíricos principais, observados na abordagem de governança aplicada às tecnologias sociais. Na sexta seção são realizadas as considerações finais, que apresentam as implicações da proposta de governança aplicada às tecnologias sociais em um contexto de desenvolvimento sustentável. Na sétima e última seção são apresentadas as referências bibliográficas.

\section{Fundamentos Teóricos}

Esta seção compreende a revisão de literatura realizada, na qual foram definidos os elementos fundamentais da governança aplicada às tecnologias sociais para populações em situação de risco socioeconômico, associada ao paradigma da sustentabilidade, que compreende os seguintes eixos: i) Governança; ii) Tecnologias Sociais, abordando a literatura sobre o conceito adotado no Brasil; e iii) Abordagem Multinível de Geels e Kemp (2007). Estes elementos da revisão são apresentados a seguir nesta ordem.

\subsection{Governança}

A governança é um tema essencial para atender às novas demandas dos arranjos constituídos em sociedades globalizadas, interconectadas e que avançam em um ritmo veloz de desenvolvimento econômico e social. Neste sentido, a governança tende a assumir uma notável função complementar aos estados e governos, principalmente quando inserida na perspectiva de assegurar a estruturação, a implementação e o desenvolvimento efetivo de tecnologias sociais sustentáveis dentro de um contexto colaborativo.

Governança, bem como governo, tem pelo menos quatro significados na literatura. Pode representar uma estrutura, um processo, um mecanismo ou uma estratégia (Faur-Levi, 2012). O conceito faz parte de uma questão fundamental para qualquer sociedade, no sentido de como ela pode governar-se com eficácia e de forma aberta (Peters, 2012). Conforme Dowbor (2009), o conceito é difícil de traduzir, uma vez que o termo governança já foi incorporado ao nosso vocabulário, implicando que, no espaço público, a boa gestão se consegue por meio da articulação inteligente e equilibrada do conjunto dos atores interessados no desenvolvimento, os chamados stakeholders.

Ao explorar as diferentes perspectivas do sentido do termo, Faur-Levi (2012) entende que governança pode assumir diversas denominações: Um chavão, um modismo, um enquadramento, um conceito ponte, um conceito guarda-chuva, um conceito descritivo, um conceito enganoso, um significante vazio, uma palavra traiçoeira, um fetiche, um campo, uma abordagem, uma teoria ou uma perspectiva. A alteração no significado de governo, a partir da incorporação ou alteração de processos, compreendendo também novas condições nas regras ou adoção de novos métodos utilizados para o governo da sociedade, pode ser tratado dentro do conceito de governança (Rhodes, 2012). A governança pode assumir um sentido mais amplo quando se refere à capacidade das pessoas e organizações de determinarem a direção e o desenvolvimento da sociedade, a partir de um ambiente democrático, com seus instrumentos e sistemas, estabelecendo condições de controle sobre políticas públicas e sobre a dinâmica econômica (Born, 2012).

O conceito de governança é relativamente novo. Só na década de 1990 a governança se tornou um conceito empolgante, segundo Faur-Levi (2012). Para o autor, uma das razões que fez da governança um conceito importante nas Ciências Sociais é o fato de que ela carrega imagens e significados relacionados a mudança e movimento. Neste sentido, esses movimentos sugerem que a autoridade é institucionalizada, ou pelo menos pode ser institucionalizada em diferentes esferas, podendo competir, negociar, coordenar entre si ou ignorar o outro, sendo agrupados em três direções diferentes: Para cima (para o regional, transnacional, 
intergovernamental e global), para baixo (para o local, regional e metropolitano) e horizontalmente (a esfera privada e civil de autoridade).

Entre as concepções mais atuais do termo, destaca-se a "boa governança", sob o ponto de vista da idealização do desenvolvimento, que ganhou o rótulo de remédio para a cura de muitos sofrimentos sociais em todo o mundo. Entretanto, nos últimos anos, a boa governança tornou-se cada vez mais importante e várias razões foram acrescentadas a sua agenda. Por isso, há uma lista de qualidades recomendadas para a boa governança, tais como equidade, participação, inclusão, democracia, entrega de serviços, regulação, descentralização, regime de comércio aberto, respeito aos direitos humanos e às diferenças de gênero, igualdade racial, um clima favorável ao investimento, uso sustentável de energia, segurança cidadã, geração de emprego e uma variedade de outros fins (Grindle, 2017).

Lynn (2012) levanta dois enfoques divergentes que podem ser discernidos para redefinir a governança. Um deles visualiza o governo e o setor orientado ao lucro como algo integral, embora não necessariamente dominante, participantes na "nova governança", ou seja, governança com o governo. O outro enfoque em torno da "nova governança”, com o fornecimento de direção societal, principalmente por parte de atores nãogovernamentais, compreenderia a governança, tornando-se centrada na sociedade civil. O primeiro enfatiza a adaptação, o último, a transformação.

As sociedades exigem escolhas coletivas sobre questões que, devido ao seu alcance, não podem ser abordadas de forma adequada pela ação individual e, por isso, alguns meios devem ser encontrados para fazer e implementar essas decisões. A necessidade dessas decisões coletivas tornou-se ainda mais evidente quando o mundo, como um todo, bem como as sociedades individuais, foram confrontados com desafios como as alterações climáticas e o esgotamento de recursos. Estes não podem ser resolvidos por ações individuais e, de fato, muitas vezes, são casos que, por interesse próprio, provavelmente resultam em dano coletivo (Hardin, 1977; Ostrom, 1990 como citado em Peters, 2012, p.).

A governança não é mais marginal ao discurso acadêmico das Ciências Sociais, enquanto adapta-se aos novos contextos culturais e institucionais, pois o tema se consolida à medida que avança em seus diferentes contextos de aplicação (Faur-Levi, 2012). Faur-Levi (2012) entende que, neste cenário, duas perspectivas colaboram para o avanço da importância do assunto: Os sistemas de governança compreendidos como estratégia e a dinâmica de governança compreendida como processo.

Em contraste, os sistemas políticos de todos os tipos são combinações complexas de demandas nas quais uma multiplicidade de fatores e condições deve ser levada em conta. Os processos sociais são diferenciados e agrupados com base em vários mecanismos, processos e forças sociais. Nesta perspectiva, a ordem não pode ser explicada apenas por uma estrutura de governança única ou pela lógica do desenvolvimento, pois há também ordens espontâneas que surgem devido à interação adaptativa em nível micro, em que os efeitos socioeconômicos se desdobram a partir das estratégias políticas ou dos esforços locais. Sistemas se tornam adaptáveis e se movem em processos ordenados quando os agentes operam de forma independente, em resposta às necessidades individuais, pressões ambientais e ações de outros (Schneider, 2012).

Um outro aspecto central a ser observado para a governança, considerando sua aplicação em tecnologias sociais, é o empoderamento, parte central de qualquer orientação participativa. A literatura sobre o empoderamento está relacionada aos métodos de alfabetização preconizados por Paulo Freire e à experiência dos sistemas de avaliação rural. Ela é centrada no ser humano e defende o diálogo como uma precondição para o aprendizado. Há uma ligação íntima entre empoderamento e apropriação, ambos conceitos estão baseados na necessidade dos recebedores de estar no coração do processo de desenvolvimento. Entretanto, empoderamento tem a ver com o aumento das capacidades (Lopes, 2005).

Santos, Coelho e Santos (2005) consideram as estruturas e a qualidade da governança determinantes críticas da coesão ou do conflito social, do sucesso ou da falha do desenvolvimento econômico, da preservação ou da deterioração do meio ambiente, bem como do respeito ou violação dos direitos humanos e das liberdades fundamentais. Os autores ainda complementam que estas considerações se estendem além da 
governança e da tecnologia e incluem considerações práticas de como manter o controle da sociedade sobre a tecnologia, bem como as instituições, estruturas e processos criados para maximizar os benefícios da tecnologia e minimizar seus riscos.

Neste cenário, Bizelli (2009) percebe um aprofundamento dos confrontos sobre a gestão das novas Tecnologias de Informação e Comunicação (TICs) e entende esses efeitos não só por serem considerados um fator estratégico para a garantia do direito à inclusão digital, mas por criarem um novo paradigma de relacionamento entre governo e sociedade, permitindo a transparência pública, publicizando o acesso do cidadão aos equipamentos e serviços da cidade e engendrando novos modelos de accountability para os agentes públicos, políticos ou não.

A governança tem de incorporar o princípio da transparência nas ações públicas para que o cidadão tenha condições de reconhecer a efetividade das políticas públicas aplicadas em prol de sua comunidade. Somente a capacidade de governança poderá permitir que o cidadão possa usufruir seus direitos constitucionais, fortalecendo, desta forma, sua confiança nos processos democráticos e nos agentes políticos que governam (Bizelli, 2009).

Há autores que desenvolveram abordagens que podem ser utilizadas no momento em que se busca entender pressupostos convergentes às tecnologias sociais. Ansell (2012) adota uma perspectiva que considera a governança colaborativa. Esse conceito é uma estratégia usada no planejamento, na regulação, na formulação de políticas e na gestão pública para coordenar, julgar e integrar os objetivos e interesses das várias partes interessadas. Conforme o autor, governança colaborativa é uma técnica usada para resolver conflitos e facilitar a cooperação entre os órgãos públicos, os interesses de grupos e os cidadãos. Ainda segundo Ansell (2012), definições de governança colaborativa podem variar ao longo de uma série de dimensões, incluindo (1) quem colabora; (2) quem patrocina a colaboração; (3) o que o termo colaboração significa; e (4) como a colaboração é organizada.

\subsection{Tecnologias Sociais}

O entendimento adotado para a criação, desenvolvimento e implantação das tecnologias sociais, neste estudo, passa, inicialmente, por uma breve análise da tecnologia apropriada e pela Abordagem Sociotécnica. Essa abordagem apresenta um aparato conceitual apropriado para descrever o processo de mudança, não somente entre as tecnologias convencionais e as tecnologias sociais, mas também entre as relações sociais associadas aos processos de mudança sobre um contexto construtivista.

Primeiramente, é necessária uma rápida análise sobre a tecnologia apropriada. A tecnologia apropriada está ligada ao marco analítico-conceitual, construído ao longo dos últimos trinta anos, que desencadeou, como uma das vertentes, a elaboração do conceito de tecnologias sociais surgido no Brasil. As abordagens para a construção da tecnologia social partem dos dois seguintes pontos, essencialmente: i) Opor-se à tecnologia convencional; e ii) incorporar a trajetória da formação do conceito de tecnologia apropriada. A tecnologia apropriada, caracterizada por atender demandas sociais e emergir de comunidades, como alternativa à corrente tecnológica tradicional, perde a importância, nos anos 1980, de constituir um modelo alternativo para o desenvolvimento, nos países menos desenvolvidos, em decorrência da ascensão do neoliberalismo e pelas limitações relacionadas ao foco em produto e na orientação normativa.

A Abordagem Sociotécnica e, em especial, o processo de construção sociotécnica, conforme Dagnino, Brandão e Novaes (2004), tem significativa importância para conceber exitosos processos de desenvolvimento de tecnologias sociais. Segundo os autores, ao romper com a visão estática e normativa de produto previamente especificado, característica da tecnologia apropriada, e ao enfatizar a necessidade de começar um processo nas condições apresentadas pelo ambiente onde ele terá de ocorrer, a adequação sociotécnica confere ao marco da tecnologia social maior solidez e eficácia. 
Nesse novo contexto de construção sociotécnica, as tecnologias sociais passam a ser uma nova modalidade de interpretação das tecnologias apropriadas, no sentido de ser uma versão mais elaborada e atualizada para enfrentar os novos desafios envolvidos na promoção de processos de desenvolvimento e de inclusão social (Novaes \& Dias, 2009).

A etapa de observação empírica é requisito para a construção do conceito de tecnologia social e sua assimilação, pois compreende o início do entendimento da dinâmica de uma sociedade em que as considerações sociológicas e técnicas estariam vinculadas. Assim, identificar e "seguir" os grupos sociais prioritários, envolvidos na construção de um artefato, é o início das pesquisas realizadas pela abordagem do contexto, que consideraram a possibilidade de a tecnologia ser uma construção social e não fruto de um processo autônomo, endógeno e inexorável, como concebe o determinismo (Dagnino, Brandão, \& Novaes, 2004).

Outro ponto central a ser considerado no contexto das tecnologias sociais que influenciam a dinâmica tecnológica tradicional é a corrente construtivista, convergente à Abordagem Sociotécnica. $\mathrm{O}$ construtivismo, ao considerar conflito e negociação entre grupos sociais, com concepções diferentes acerca dos problemas e soluções no desenvolvimento tecnológico, questiona a visão da dinâmica tecnológica. A partir desse debate, o arranjo estrutural de cada circuito ou programa é renovado por uma configuração social específica, que serve de unidade e escolha (Dagnino et al., 2004).

Logo, os autores Dagnino et al. (2004) apontam que as tecnologias empregadas seriam selecionadas segundo um processo pautado pelos códigos sociotécnicos, definidos pela correlação de forças sociais e políticas, que delimitam o espaço de sua consolidação. Assim, os conceitos apresentados permitem entender porque a tecnologia, uma vez estabelecida ou "fechada", passa a aceitar esses códigos sociotécnicos.

Assim como Dagnino et al. (2004), Valadão, Andrade e Cordeiro (2014) concordam sobre um ponto relevante a ser considerado nessas perspectivas sociotécnicas, que é o fato de a tecnologia não se restringir aos artefatos materiais, que são socialmente definidos e produzidos, nem se prender apenas à relação com as pessoas envolvidas, como é usualmente discutido.

Ainda no contexto da preocupação com a tecnologia social, Dagnino et al. (2004) destacam que a adequação sociotécnica teria por objetivo adequar a tecnologia convencional (e, inclusive, conceber alternativas), aplicando critérios suplementares aos técnico-econômicos usuais, a processos de produção e circulação de bens e serviços em circuitos não-formais, situados em áreas rurais e urbanas, visando aperfeiçoar suas implicações.

Segundo Baumgarten (2008), acredita-se que as tecnologias sociais teriam a potencialidade para expressar instâncias físicas e virtuais de trocas, realizar reintegração de saberes e estabelecer trocas intercampos e interdisciplinas, que se fazem por sendas, através das quais se vem construindo conhecimentos que dão conta da complexidade do mundo real e de nossas capacidades para construí-lo e reconstruí-lo, de acordo com nossas necessidades e potencialidades.

Dagnino (2004) convida à reflexão acerca do tema com a seguinte pergunta crucial: Por que é necessário conceber tecnologia social? $\mathrm{O}$ autor argumenta que essa pergunta pode ser respondida considerando duas razões fundamentais e exigindo um processo de sensibilização das organizações, do estado e da sociedade. Primeiro, porque considera-se que a tecnologia convencional não é adequada para a inclusão social. Segundo, porque entende-se que as instituições públicas envolvidas com a geração de conhecimento científico e tecnológico não parecem estar em um nível de capacitação para desenvolver uma tecnologia capaz de viabilizar a inclusão social e oferecer autossustentabilidade aos empreendimentos autogestionários que ela deverá alavancar.

É essencial considerar também para o contexto brasileiro o conceito desenvolvido pelo Instituto de Tecnologia Social (ITS) para a tecnologia social, fundamentado em um conjunto de técnicas e metodologias transformadoras, desenvolvidas e (ou) aplicadas na interação com a população e apropriadas por ela, que representam soluções para inclusão social e melhoria das condições de vida (ITS, 2004). 
A preocupação com o processo de produção da tecnologia social, embora não prescinda de aspectos gerenciais, volta-se, prioritariamente, para a emancipação dos atores envolvidos, tendo no centro os próprios produtores e usuários dessas tecnologias. A tecnologia social implica a construção de soluções de modo coletivo pelos que irão se beneficiar dessas soluções e que atuam com autonomia, ou seja, não são apenas usuários de soluções importadas ou produzidas por equipes especialistas, a exemplo de muitas propostas das diferentes correntes da tecnologia apropriada (Rodrigues \& Barbieri, 2008).

Jesus e Bagattolli (2013) apresentam uma importante aplicação presente nas tecnologias sociais, que é o aspecto de integração. Segundo as autoras, os resultados e os aprendizados práticos indicam que o trabalho com tecnologia social pode lograr impacto ainda mais interessante caso se utilize uma estratégia de integração de tecnologias, ou seja, um olhar para os diferentes problemas e potencialidades de uma localidade, de forma integrada, e uma intervenção com diferentes tipos de tecnologia social.

Quanto às experiências de tecnologia social, foi observado um crescimento significativo entre 2007 e 2012. A princípio, parecia ser uma contradição um aumento desse tipo de iniciativa, afinal, como uma tecnologia pode ser "social", se toda tecnologia surge na sociedade? Mas seu entendimento transcende a confusão semântica e passa a ser compreendido como tecnologia para inclusão social. A tecnologia social também se fortalece quando passa a ser incorporada por agendas de instituições governamentais, transformando-se em ações por meio de políticas públicas (Jesus \& Bagattolli, 2013).

Assim, a tecnologia social se fortaleceu nos últimos anos como uma estratégia para a inclusão socioeconômica, propondo-se a estimular uma nova lógica para o desenvolvimento tecnológico, conforme apontam Jesus e Bagattolli (2013). As autoras mencionam que, embora as entidades que se dedicam a Ciência, Tecnologia e Inovação (CT\&I) não se envolvam com o debate, relevantes instituições públicas estão desenhando suas ações baseadas na reaplicação de tecnologia social, buscando dar amplitude à proposta e articulando positivamente atores sociais de diferentes naturezas.

\subsection{Abordagem Multinível}

A abordagem de governança voltada às tecnologias sociais elaborada no estudo contemplou uma sustentação teórica fundamentada na Abordagem Sociotécnica, em conjunto com a perspectiva multinível, seguindo uma linha estruturalista que observa uma interdependência dos elementos para a formação da organização e entendimento das dinâmicas entre eles.

A abordagem de governança voltada às tecnologias sociais é, em si mesma, uma perspectiva que interrelaciona aspectos a serem observados de maneira integrada para a obtenção de estratégias, resultados e impactos sustentáveis. Entretanto, apenas essa abordagem, isolada de outras relações, permaneceria estática e com efeitos limitados, assim, para corrigir esta limitação, neste estudo, foi vinculada à perspectiva multinível de Geels e Kemp (2007). A partir desse vínculo, foi possível realizar uma fundamentação para um melhor entendimento dos espaços a serem ocupados pelos elementos da governança voltada às tecnologias sociais, assim como uma melhor compreensão das dinâmicas, mudanças e transições geradas ao longo do tempo, a fim de corrigir distorções de desenvolvimento presentes nas populações em situação de vulnerabilidade socioeconômica.

As perspectivas, adotadas de forma integrada, oferecem um referencial apropriado aos elementos da governança, a serem tratados nessa abordagem, envolvendo um contexto de complexidade constituído pelos regimes político, tecnológico, científico, sociotécnico e cultural. Isso inclui diversos atores de grupos sociais distintos, tais como governo, organizações não governamentais e empresas, todos envolvidos no processo de transição para uma nova etapa que incorpora a governança em tecnologias sociais visando a sustentabilidade de comunidades vulneráveis no Brasil.

É necessário destacar que a presente abordagem não tem como objetivo simplificar uma realidade de dimensões complexas e de difícil contextualização, com risco de ensejar um teor reducionista. A construção da 
governança em tecnologias sociais, em seus elementos e efeitos envolvidos nas transições para novas etapas que ofereçam atributos mais qualificados e transparentes para a sustentabilidade das tecnologias sociais, criadas e implementadas em comunidades em situação de vulnerabilidade socioeconômica, objetiva potencializar capacidades dos atores e reduzir riscos ao desenvolvimento de longo prazo das comunidades, tais como corrupção, falta de planejamento, ausência de apoio técnico sistêmico, desequilíbrio político, entre outros.

Nesse sentido, Geels e Kemp (2007) apresentam um olhar que incorpora estabilidade e mudança sobre o nível sistêmico. Os autores tratam sobre as dinâmicas em sistemas sociotécnicos, padrões de mudança do sistema e desenvolvem, a partir deste contexto, uma tipologia na perspectiva multinível que compreende os três seguintes tipos de mudança: i) Reprodução; ii) transformação; e iii) transição, que foram adotadas para a construção da abordagem. Observa-se que, originalmente, a perspectiva multinível foi desenvolvida para o entendimento das transições e de mudanças nos regimes, que decorrem da sociologia da tecnologia, na qual três dimensões inter-relacionadas são importantes: (a) Os sistemas sociotécnicos, como elementos tangíveis necessários para cumprir funções sociais; (b) os grupos sociais, que mantêm e refinam os elementos dos sistemas sociotécnicos; e (c) regras (entendidas como regimes) que guiam e orientam as atividades de grupos sociais.

O conceito de regime foi ampliado para "regimes sociotécnicos", conforme cita Geels (2004), os quais incluem cientistas, usuários, decisores políticos e grupos sociais, além de engenheiros e empresas, os quais interagem e formam redes interdependentes, resultando no alinhamento de atividades. Esta coordenação intergrupo é representada com o conceito de regimes sociotécnicos.

O regime sociotécnico constitui o nível meso na perspectiva multinível. Uma característica a ser ressaltada é que os regimes sociotécnicos são responsáveis pela estabilidade dos sistemas sociotécnicos existentes. Por muitas razões, os sistemas sociotécnicos existentes são caracterizados pela estabilidade, sendo que grande parte da mudança observada é de um tipo de reprodução na qual o sistema sociotécnico está sendo reproduzido, conforme apontam Geels e Kemp (2007).

Para que um estágio de estabilidade de um sistema seja alterado, há necessidade de mudança, que pode ocorrer de diversas maneiras. Especificamente, as mudanças ocorrem no âmbito dos nichos, no nível micro, os quais guardam a essência das inovações que irão surgir, de tal forma que o desenvolvimento nos sistemas possa vir a ocorrer. Além disso, os nichos resguardam-se de ações do mercado, emergindo características próprias de seus contextos sociotécnicos, podendo ocorrer de maneira isolada ou em rede, segundo Geels e Kemp (2007).

Neste entendimento, os nichos podem ser vistos como um fenômeno criado em nível micro, interagindo com os regimes estabelecidos no nível meso, dentro de um panorama macro. Consequentemente, em nível macro, de acordo com Geels e Kemp (2007), forma-se o panorama sociotécnico, que se refere a aspectos do ambiente exógeno que está além da influência direta dos atores. O conteúdo do panorama sociotécnico é heterogêneo e pode incluir aspectos como o crescimento econômico, amplas coalizões políticas, os valores culturais e normativos, e os problemas ambientais e de escassez de recursos. Diante deste contexto, no que tange a mudanças, observa-se que o panorama material muda muito lentamente. Por outro lado, o cenário político é mais dinâmico, no qual poderemos testemunhar revoluções, novas coalizões e novas ideias, criando um espaço para a inovação e as mudanças no sistema.

Diante da dinâmica e da alta responsabilidade sobre os aspectos de planejamento, a compreensão por parte dos responsáveis pelas políticas públicas, com relação a como estruturar melhor as estratégias e os processos orientados aos resultados da sustentabilidade no nível de sistema, se faz necessária no que concerne ao uso de energia, recursos, combate ao desperdício e respectivos desdobramentos sobre o desenvolvimento que se espera (Evans et al., 2017).

O processo de transição no panorama sociotécnico, gerado pela mudança e pautado por premissas sustentáveis, é um aspecto determinante para os impactos no longo prazo. Estratégias, recursos e as competências de atores influenciam nos resultados e impactos esperados, estabelecendo mudanças e retroalimentando o nível de sistema a partir das açóes implementadas, gerando transições. Assim, as 
mudanças que impulsionam as transições nos sistemas sociotécnicos podem ser definidas estrategicamente por atores com um plano maior e visão de futuro, até determinada medida (Farla, Markard, Raven, \& Coenen, 2012).

No eixo das expectativas estratégicas da sustentabilidade no longo prazo, Smith, Stirling e Berkhout (2005) apontam uma perspectiva da governança a partir de transições sociotécnicas sustentáveis, incorporando os elementos agência e poder no processo de transformação do regime, em um modelo quasi-evolucionário, no qual regimes enfrentam pressões de seleção de forma contínua. O regime é entendido como um sistema sujeito a pressões de seleção e é capaz de adaptar-se a estas pressões. Já a governança é entendida tanto como a sustentação de contextos de transição, quanto como um conjunto de intervenções que visam alterar tais contextos.

Contextos de transição diferenciados, conforme argumentam Smith et al. (2005), determinam a forma e a direção da mudança de regime em resposta a essas pressões de seleção. A articulação de pressões e o grau em que as respostas são coordenadas, com base nos recursos disponíveis no âmbito do regime, definem o contexto de transição. O poder para afetar a mudança depende de adesão ao regime e da distribuição de recursos para a mudança das expectativas.

No entanto, segundo o entendimento de Geels (2011), existem problemas mais profundos para as teorias do processo de tópicos multifacetados no que tange à metodologia. $\mathrm{O}$ autor destaca que a perspectiva multinível não deve ser reduzida a um procedimento mecânico, forçando-a em uma camisa de força. Isso se deve ao fato de que a pesquisa de fenômenos complexos, como as transições, não pode ser reduzida com a aplicação de procedimentos metodológicos e sempre vai conter elementos de interpretação criativa, a fim de atender novas curvas de complexidade inseridas em novas dinâmicas com elementos agrupados, existentes ou novos.

\section{Procedimentos Metodológicos}

O presente estudo foi elaborado a partir de uma abordagem qualitativa, de cunho exploratório, no sentido de estabelecer os parâmetros iniciais para a elaboração da abordagem de governança em tecnologias sociais. Esta estrutura foi definida para possibilitar um melhor entendimento sobre os aspectos iniciais relacionados à abordagem, a fim de identificar evidências, experiências, resultados, modelos e aspectos críticos, de forma que se pudesse estabelecer um cenário preliminar sobre a formação do tema.

O método adotado para a elaboração desta pesquisa compreendeu as etapas de coleta e análise de dados.

A etapa de coleta de dados compreendeu, primeiramente, a coleta de dados secundários, na qual foram analisados sites e documentos, a fim de verificar as instituiçóes que integraram a amostra da pesquisa e as informações que subsidiaram a elaboração do roteiro semiestruturado, utilizado nas entrevistas com as instituições escolhidas. Posteriormente, houve a coleta de dados primários, que compreendeu a realização de entrevistas com dirigentes das instituições-chaves, voltadas para a tecnologia social em diferentes estados brasileiros.

A coleta de dados primários ocorreu por meio de entrevistas semiestruturadas, através de abordagem direta, com dirigentes de instituições reconhecidas por atuarem com tecnologias sociais no Brasil, envolvendo os dois seguintes segmentos:

- Instituições que formulam as políticas públicas que, por sua vez, influenciam modelos de governança em tecnologias sociais;

- Instituições que adotam a governança em tecnologias sociais para cumprirem seus objetivos institucionais.

Quanto à operacionalização desta pesquisa, as entrevistas foram realizadas de maneira presencial, conforme descrição a seguir. 
Figura 1. Grupo das instituições que formulam as políticas públicas.

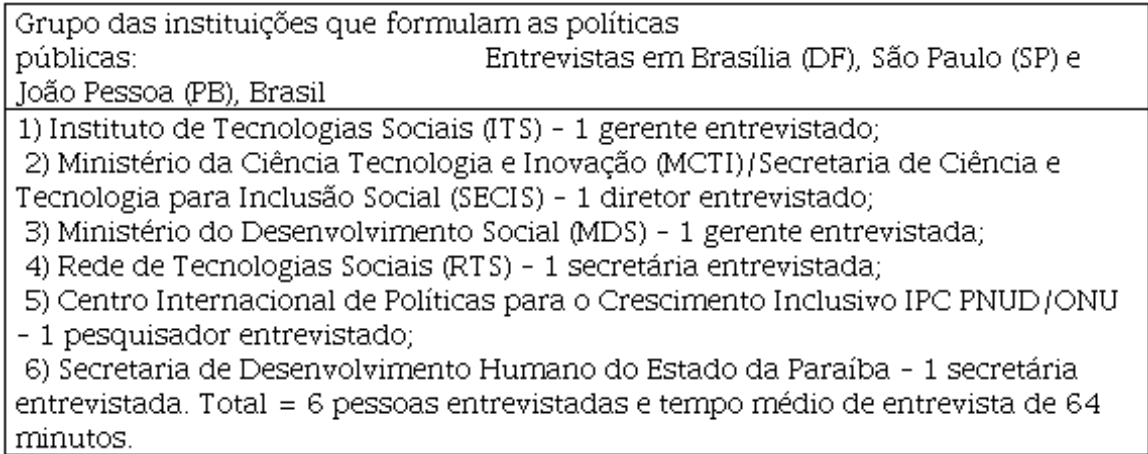

Figura 2. Grupo das instituições que adotam a governança em tecnologias sociais.

Grupo das instituições que adotam a governança em tecnologias sociais:
Entrevistas em São Paulo (SP), João Pessoa (PB), Teixeira (PB) e Porto Alegre (RS),
Brasil
1) Instituto de Tecnologia Social (ITS) - 1 gerente entrevistado;
2) Caos Focado - 1 diretor entrevistado;
3) Cunhã Coletivo Feminista - 1 diretora entrevistada;
4) Centro de Educação Popular e Formação Social (CEPFS) - 1 diretor entrevistado;
5) Instituto para o Desenvolvimento de Energias Alternativas e da Auto
Sustentabilidade (IDEAAS) - 1 diretor entrevistado;
6) Turma do Bem - 1 diretor entrevistado;
7) Projeto Centro de Integração de Educação e Saúde (CIES) - 1 gerente
entrevistado. Total = 7 pessoas entrevistadas e tempo médio de entrevista de 63
minutos.

A amostra foi definida em função do critério "nível de maturidade da instituição", utilizado para determinar a característica inicial das instituições participantes que adotam a governança em tecnologias sociais. Logo, as instituiçóes integrantes da amostra atenderam aos critérios referentes a tecnologias sociais, validadas e implantadas em sua região, em atividade há mais de 2 anos, tendo obtido resultados expressivos em suas regiões de atuação. A base de dados para a identificação das instituições foi a da Fundação do Banco do Brasil, uma das mais completas disponíveis no país, que compreende um banco de Tecnologias Sociais, com informações sobre as tecnologias sociais certificadas no âmbito do Prêmio Fundação Banco do Brasil de Tecnologia Social.

Já a escolha das instituições que formulam as políticas públicas teve como foco o Distrito Federal e a cidade de João Pessoa, capital da Paraíba. A primeira, em função de ser o centro das políticas públicas, com impacto em nível nacional em relação às tecnologias sociais. A segunda, em função do governo de um estado com Índice de Desenvolvimento Humano Municipal (IDHM) baixo, na época do estudo, tendo ocorrido reeleição para o governo estadual, que possibilitou um acompanhamento mais sólido, em decorrência da continuidade das equipes atuantes e de políticas públicas voltadas para a populações em situação de vulnerabilidade socioeconômica.

A parte da análise buscou apresentar, primeiramente, as informações gerais obtidas via entrevistas semiestruturadas sobre as instituições de tecnologias sociais no Brasil. Em um segundo momento, foram apresentados os dois grupos de instituições que participaram da pesquisa deste estudo, as instituições formuladoras de políticas públicas em tecnologias sociais no Brasil e as instituições que adotam governança em tecnologias sociais no Brasil. Posteriormente, foram identificadas as categorias mais relevantes, à luz 
da revisão de literatura, que contribuíram para a formulação das premissas iniciais sobre a proposta de governança aplicada às tecnologias sociais para populações em situação de risco socioeconômico associada ao paradigma da sustentabilidade.

A próxima seção abordará os resultados obtidos e os aspectos que compreendem a nova perspectiva de impactos, a partir da adoção de governança em tecnologias sociais para a sustentabilidade em populações em situação de risco socioeconômico no Brasil.

\section{Resultados}

A abordagem de governança aplicada às tecnologias sociais surgiu a partir da análise de informações obtidas de instituições que elaboram as políticas públicas no Brasil sobre Tecnologias Sociais e de instituições que atuam com Tecnologias Sociais no Brasil.

A premissa da abordagem, que considerou os dois grupos de instituições, possibilitou avaliar os elementos fundamentais da governança aplicada às tecnologias sociais que surgiram na pesquisa. $O$ resultado desta não se limitou a encerrar a discussão, apontando uma única forma, mas sim, buscou indicar os pontos de partida para o tratamento de uma governança para as tecnologias sociais se estabelecerem no Brasil, como um vetor de desenvolvimento sustentável e motor da economia para alavancar comunidades em situação vulnerável.

Nesse contexto, foi possível identificar as estratégias definidas por instituições governamentais e a resposta das ações e projetos desenvolvidos pelas instituições voltadas às tecnologias sociais. Uma dinâmica inicial foi obtida no sentido de entender como a estratégia é elaborada no nível meso e afeta o desenvolvimento das comunidades vulneráveis nos nichos (Geels \& Kemp, 2007), e como as instituições que atuam com tecnologias sociais reagem ou interagem para obter as transformações socioeconômicas necessárias para as populações-alvo.

Uma parte da abordagem, resultante da pesquisa, contempla os tipos de governança identificados nas instituições que adotam governança em tecnologias sociais no Brasil. Os resultados obtidos compreendem uma proposta nova, ainda não verificada na literatura, voltada a identificar, primeiramente, parâmetros de governança em tecnologias sociais em instituições brasileiras e, após essa identificação, promover novas pesquisas a fim de avaliar os tipos de governança que puderam ser observados.

Neste sentido, como não há literatura para relacionar a este caso, o procedimento de análise adotado foi o de identificar as ocorrências sobre os principais processos relacionados à governança presentes nas organizações avaliadas. A partir das ocorrências, foi obtido um quadro com os principais resultados, em uma proposta para definição de níveis de governança, identificados nas Instituições que Adotam Governança em Tecnologias Sociais no Brasil. 
Figura 3. Tipos de governança identificados nas instituições que adotam governança em tecnologias sociais no Brasil.

\begin{tabular}{|c|}
\hline $\begin{array}{l}\text { ipos de Governança Identificados nas Instituições que Adotam Governança em } \\
\text { ecnologias Sociais no Brasil }\end{array}$ \\
\hline $\begin{array}{l}\text { Não Estabelecida (NE) } \quad \text { Ainda não há aspectos de governança observados na } \\
\text { instituição. }\end{array}$ \\
\hline $\begin{array}{l}\text { Estágio Inicial (EI) } \\
\quad \text { Desenvolvendo a cultura institucional para recepcionar as práticas de } \\
\text { governança em tecnologias sociais; } \\
\quad \text { Instituição possui conhecimentos básicos de gestão aplicados a negócios } \\
\text { tradicionais; } \\
\text { Início da criação das diretrizes institucionais voltadas à governança em } \\
\text { tecnologias sociais. }\end{array}$ \\
\hline $\begin{array}{l}\text { Estágio Intermediário (EINT) } \\
\quad \text { Cultura institucional preparada para recepcionar as práticas de governança } \\
\text { em tecnologias sociais; } \\
\quad \text { Instituição possui conhecimentos intermediários de gestão aplicados a } \\
\text { negócios tradicionais e avança no aprimoramento de conhecimentos específicos } \\
\text { voltados à gestão aplicada às tecnologias sociais; } \\
\quad \text { Diretrizes institucionais voltadas à governança em tecnologias sociais } \\
\text { estabelecidas e implantadas. }\end{array}$ \\
\hline $\begin{array}{l}\text { Estágio Avançado (EA) } \\
\quad \text { Cultura institucional está na fase de aprimoramento em relação às práticas } \\
\text { de governança em tecnologias sociais; } \\
\quad \text { Instituição possui conhecimentos avançados de gestão, aplicados a negócios } \\
\text { tradicionais, aceitos internacionalmente, e elabora conhecimentos específicos, } \\
\text { voltados à gestão aplicada às tecnologias sociais; } \\
\quad \text { Diretrizes institucionais voltadas à governança em tecnologias sociais, } \\
\text { implantadas e em aprimoramento. }\end{array}$ \\
\hline
\end{tabular}

Já os impactos, a partir da adoção de governança em tecnologias sociais, que compreendem vocação regional, inclusão social e políticas públicas, estão voltados às transformações sustentáveis esperadas a serem efetivadas nas comunidades vulneráveis, para a geração de um novo patamar de sustentabilidade, com melhor qualidade de vida para as populações beneficiárias.

Os resultados obtidos são uma novidade para o tema de governança, pois estão relacionados a uma proposta ainda não verificada na literatura, relacionada a parâmetros de governança em tecnologias sociais em instituições brasileiras. Da mesma forma, como não há literatura para relacionar a este caso, dos possíveis impactos do desenvolvimento sustentável em populações em situação de risco social e econômico no Brasil, o procedimento de análise adotado foi o de identificar as ocorrências relevantes para o tema, a partir das perguntas que integraram o roteiro para entrevistas, envolvendo instituiçóes que adotam governança em tecnologias sociais e instituições formuladoras de políticas públicas em tecnologias sociais.

A partir das ocorrências, desenvolveu-se dois quadros apresentando uma comparação entre uma perspectiva sem governança em tecnologias sociais e uma perspectiva com governança em tecnologias sociais. Esta última faz parte da proposta que considera uma nova perspectiva de impactos a serem incluídos na pauta de governança em tecnologias sociais, que deverão ser observados em uma nova etapa de desenvolvimento, integrando possíveis estratégias e finalidades para a abordagem de governança aplicada às tecnologias sociais no Brasil. 

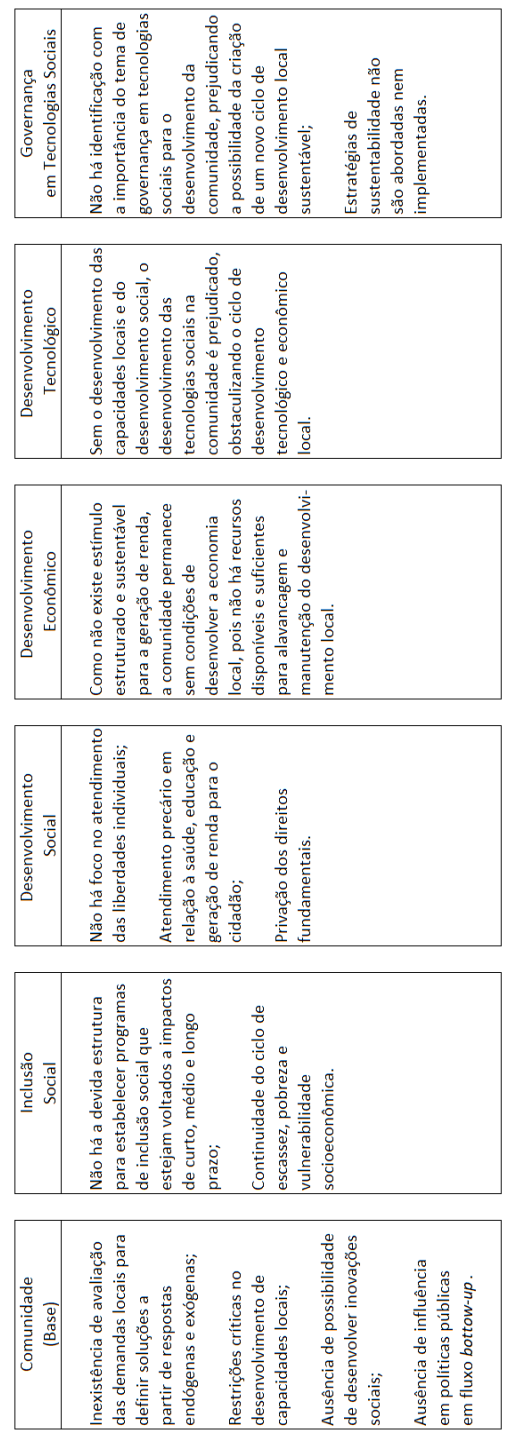

Figura 4. Perspectiva de impactos sem a adoção de governança em tecnologias sociais para a sustentabilidade em populações em situação de risco socioeconômico no Brasil. 


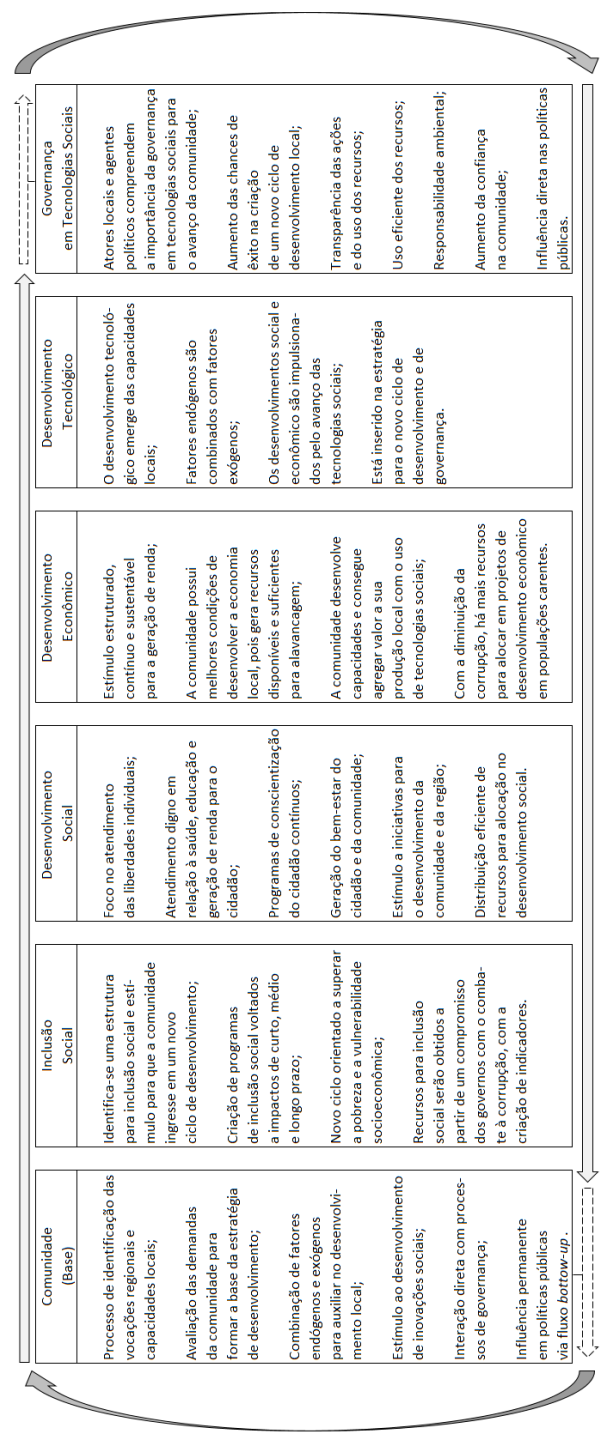

Figura 5. Nova perspectiva de impactos a partir da adoção de governança em tecnologias sociais para a sustentabilidade em populações em situação de risco socioeconômico no Brasil.

Neste cenário, a abordagem de governança aplicada às tecnologias sociais passa a compreender um conjunto de elementos que emergiram a partir de uma análise inicial sobre o contexto das políticas públicas e das instituições que atuam com tecnologias sociais no Brasil, voltados a um desenvolvimento de longo prazo mais amplo e responsável, observando impactos sustentáveis a serem verificados nas populações alvo.

Os elementos que compõem a abordagem de governança aplicada às tecnologias sociais são apresentados a seguir.

Panorama Sociotécnico

- Transformações verificadas nas populações vulneráveis em relação à melhoria do âmbito socioeconômico, gerando novos estágios e novas demandas para novas curvas de desenvolvimento;

- Políticas públicas voltadas ao estímulo à renovação dos níveis de desenvolvimento das tecnologias sociais presentes nas populações em situação de vulnerabilidade socioeconômica.

Nível Meso

- Influência nas políticas públicas regionais e nacionais através de um processo bottom-up;

- Processamento das inovações incorporadas aos nichos que exercerão pressão para novas curvas de desenvolvimento no Panorama Sociotécnico; 
- Desenvolvimento de novas dinâmicas em relação aos impactos da sustentabilidade nas populações em situação de vulnerabilidade socioeconômica;

- Intercâmbio de experiências entre instituições que atuam com tecnologias sociais para estimular novos ciclos de desenvolvimento.

Nível de Nicho

- Desenvolvimento de lideranças que entendam a importância da adoção de uma governança para assegurar a devida implantação das tecnologias sociais em populações em situação de vulnerabilidade socioeconômica;

- Compreensão das vocações regionais e locais das comunidades como base para alavancar iniciativas de desenvolvimento;

- Estímulo a inovações sociais para um melhor aproveitamento do processo de desenvolvimento das tecnologias sociais;

- Transparência e comunicação;

- Gestão e uso eficiente dos recursos disponíveis na comunidade;

- Cultura da tecnologia social incorporada na comunidade.

\section{Discussão}

Os estudos sobre tecnologias sociais já realizados estão relacionados à formação de conceitos sobre o tema (Baumgarten, 2008; Its, 2004; Rodrigues \& Barbieri, 2008; Dagnino et al., 2004). A partir das abordagens estudadas, identificou-se uma lacuna entre as tecnologias sociais e os processos de gestão relacionados, de forma a assegurar práticas sistematizadas para a obtenção de resultados e impactos mais efetivos para populações em risco social e econômico, assim como para o poder público e a sociedade.

Desafios no âmbito de novas pesquisas que contemplassem um maior número de atores e relações em processos de mudança e transições já haviam sido considerados por outros autores (Farla et al., 2012). A perspectiva proposta de relacionar outros elementos às tecnologias sociais, tais como a governança, sustentabilidade, desenvolvimento econômico, desenvolvimento social, desenvolvimento tecnológico e impactos provenientes desse contexto, integrando-os em uma abordagem que beneficie populações excluídas e em vulnerabilidade socioeconômica é um diferencial importante para a sociedade civil e os gestores públicos. O conceito abrirá precedentes para aplicações em diferentes setores, em diferentes regiões do país, assim como auxiliará a descoberta de novos estudos voltados à abordagem de governança em tecnologias sociais.

Os conceitos de governança aplicada às tecnologias sociais apresentados foram fundamentados em uma análise inicial dos conceitos tradicionais de governança. Foi adotada a perspectiva multinível de Geels e Kemp (2007) para estabelecer os fundamentos da dinâmica na qual a governança em tecnologias sociais estará inserida para promover transições sustentáveis, com novos níveis de desenvolvimento, voltadas a comunidades inseridas em situação de vulnerabilidade socioeconômica no Brasil.

Os impactos envolvidos na adoção de uma abordagem de governança em tecnologias sociais transcendem os benefícios para populações excluídas e em situação de risco do ponto de vista socioeconômico. A proposta deste estudo buscou gerar uma abordagem de governança que possa auxiliar na identificação de melhorias em áreas sociais prioritárias, no sentido de qualificar os processos para atendimento de demandas, transferir conhecimento e oferecer condições para estimular a economia e o avanço da qualidade de vida de populações vulneráveis.

Outro aspecto que sustenta a continuidade dos estudos é a avaliação dos níveis de transparência nas ações de tecnologias sociais no Brasil, a fim de coibir práticas de corrupção no uso de recursos e, com isso, gerar fundos para medir os resultados deste combate e aumentar os investimentos em projetos para o avanço de iniciativas em áreas críticas do país que necessitam de capital para evoluir.

Deve-se considerar, ainda, que o estudo em outros países da América Latina sobre a abordagem de governança em tecnologias sociais também se faz necessário. Há muitas semelhanças estruturais entre os 
países periféricos. Assim, milhões de pessoas dentro da faixa de vulnerabilidade socioeconômica teriam condições de acessar modelos que possibilitem oportunidades para ingressar em um novo ciclo de sustentabilidade para promover melhores níveis de qualidade de vida. Estudos nesses outros países são fundamentais e também podem ser realizados em cooperação com o Brasil.

Existe também um desafio muito importante, que é o de transcender identidades ideológicas e partidárias para uma transformação social que ocorre via integrações, de diferentes agentes sociais, com diferentes visões, independentemente de bandeiras. Esta observação é particularmente importante quando ocorre troca de governos com orientações partidárias distintas, que normalmente irão refletir em mudanças nas políticas econômicas e sociais.

Uma visão integrada de longo prazo, assegurada por leis, voltada aos interesses e prioridades de uma nação, podem fazer a diferença para milhóes de pessoas, desencadeando, desta forma, a possibilidade de se resgatar, muitas vezes, o equilíbrio perdido em função de uma pauta dissociada das reais demandas do país.

\section{Considerações Finais}

A abordagem de governança aplicada às tecnologias sociais, sugerida neste estudo, enseja um recurso para gestores que atuam em instituiçóes voltadas para tecnologias sociais e que atuam como formuladores de políticas públicas, pois amplia o contexto e as variáveis envolvidas nos desafios de inserir a sustentabilidade em populações em vulnerabilidade socioeconômica no país.

Os resultados obtidos nesta pesquisa representam uma nova forma de olhar a dinâmica das tecnologias sociais, problemas, inovações, modelos, estágios, transições, desafios e mudanças, a fim de imprimir uma etapa mais consistente para ações voltadas à sustentabilidade nos processos de inclusão social. Fica o convite para que pesquisadores utilizem a abordagem para futuras validações ou até mesmo para a identificação de restrições, ambas relevantes para o avanço das pesquisas sobre o tema.

Destaca-se que a perspectiva adotada neste estudo, de compreender de maneira integrada fatores de impactos entre formuladores de políticas públicas e instituições que atuam com tecnologias sociais, é muito importante e necessita de mais estudos. Além disso, ressalta-se a necessidade de se ampliar a amostra de instituições e atores, pois há diversos beneficiários, especialistas, acadêmicos e outras esferas governamentais que não foram avaliados para a compreensão mais aprofundada dos aspectos da governança em tecnologias sociais.

Entre as principais limitações desta pesquisa, pode-se destacar a dificuldade de acesso a gestores públicos, no âmbito federal, para apresentar informações sobre o tema. A amostra com poucas instituições avaliadas, de alguns estados brasileiros, com poucas pessoas, também insere-se nas restrições deste trabalho. Outra limitação verificada foi em decorrência da restrição de tempo e de recursos, ao não terem sido inseridos os beneficiários das tecnologias sociais e outros profissionais que atuam com o tema para uma investigação mais elaborada acerca dos impactos das estratégias adotadas pelas instituições que atuam com governança em tecnologias sociais e formuladores de políticas públicas em tecnologias sociais no país.

A abordagem deste estudo buscou um retrato atual acerca da governança presente nas instituições que atuam com governança em tecnologias sociais e em instituições que formulam políticas públicas em tecnologias sociais. Foi possível, por um lado, entender algumas lacunas existentes entre os fatores analisados nas instituições formuladoras de políticas públicas, tais como divergências nas avaliações de resultados, cumprimento de metas e fragilidade das políticas públicas em tecnologias sociais no Brasil. Por outro lado, foi observado que as organizações da sociedade civil que atuam com tecnologias sociais estão mais bem preparadas que as instituições do governo, em termos de gestão e governança, para assegurar um desempenho mais eficiente no cumprimento de seus objetivos organizacionais.

Por fim, o estudo realizado cumpriu com os objetivos propostos, buscou contribuir através de uma proposta integrada entre atores, processos, dinâmicas, fatores e impactos para o avanço dos estudos e dos 
debates acadêmicos em tecnologias sociais. Portanto, a governança em tecnologias sociais se apresenta como uma contribuição valiosa para acadêmicos, organizações da sociedade civil e governo em prol de uma etapa mais justa, mais transparente, mais digna e orientada à inclusão social sustentável no Brasil.

\section{REFERÊNCIAS}

Ansell, C. (2012). Collaborative Governance. In: Faur-Levi, D. Oxford Handbook of Governance. New York: University Press.

Baumgarten, M. (2008). Ciência, tecnologia e desenvolvimento - Redes e inovação social. In: Parcerias Estratégicas (pp. 101-124). Brasília: CGEE - Centro de Gestão e Estudos Estratégicos, Ministério da Ciência e Tecnologia, 26.

Bizelli, J. L. Estado, Democracia e Gestão da Inovação. (2009). In: Bizelli, J. L., \& Ferreira, D. A. O. (Orgs.). Governança Pública e Novos Arranjos de Gestão (pp. 15-34). Piracicaba: Jacintha Editores.

Born, R. H. (2012). Rio+20, Economia verde e governança do desenvolvimento sustentável: desafios associados às políticas públicas e construção de um mundo justo e saudável. In: Gerber, E., Papa, F., Tible, J., Sanseviero, R., \& Balardini, S. (Orgs.). Modelos de Desenvolvimento, Trabalho e Sustentabilidade: desafios para o progressismo na América Latina (pp. 86-106). São Paulo: Fundação Friedrich Ebert.

Dagnino, R. (2004). A tecnologia social e seus desafios. Apresentação realizada para os participantes do processo de construção da Rede de Tecnologia Social (RTS). Recuperado de: <www.ige.unicamp.br/site/publicacoes/138/ A\%20tecnologia\%20social\%20e\%20seus\%20desafios.pdf>.

Dagnino, R., Brandão, F.C., Novaes, H.T. (2004). Sobre o marco analítico conceitual da tecnologia social. In: Lassance Jr. et al. Tecnologia Social - uma estratégia para o desenvolvimento. Rio de Janeiro: Fundação Banco do Brasil.

Dowbor, L. (2009). Inovação Social e Sustentabilidade. Revista Brasileira de Gestão Urbana, Curitiba, 1(1), 109-125.

Evans, S., Vladimirova, D., Holgado, M., Van Fossen, K., Yang, M., Silva, E. A., Barlow, C. Y. (2017) Business Model Innovation for Sustainability: Towards a Unified Perspective for Creation of Sustainable Business Models. Bus. Strat. Env., 26: 597-608.

Farla, J., Markard, J., Raven, R., Coenen, L. (2012). Sustainability transitions in the making: A closer look at actors, strategies and resources. Technological Forecasting and Social Change, 79 (6), pp. 991-998.

Faur-Levi, D. (2012). From "Big Government" to "Big Governance". In: Faur-Levi, D. Oxford Handbook of Governance. New York: University Press.

Geels, F. W. (2011). The multi-level perspective on sustainability transitions: Responses to seven criticisms. Environmental Innovation and Societal Transitions (Vol.1, pp. 24-40).

Geels, F. W. (2004). From sectoral systems of innovation to socio-technical systems Insights about dynamics and change from sociology and institutional theory. Research Policy (Vol. 33, pp. 897-920).

Geels, F. W., Kemp, R. (2007). Dynamics in socio-technical systems: Typology of change processes and contrasting case studies. Technology in Society (Vol. 29, pp. 441-455).

Grindle, M. S. (2017), Good Governance, R.I.P.: A Critique and an Alternative. Governance, 30: 17-22.

Hardin, G. (1977). Managing the Commons. In: Modern governance (pp. 35-48), edited by J. Kooiman. London: Sage.

ITS, Instituto de Tecnologia Social. (2004). Reflexões sobre a construção do conceito de tecnologia social. In: Lassance et al. Tecnologia Social - uma estratégia para o desenvolvimento. Rio de Janeiro: Fundação Banco do Brasil.

Jesus, V. M. B., \& Bagattolli, C. (2013). Integração de tecnologias sociais: reflexões sobre práticas iniciais. In: Costa, A. B. (Org.). Tecnologia Social e Políticas Públicas. Brasília: Fundação Banco do Brasil.

Lopes, Carlos. (2005). Cooperação e desenvolvimento humano: a agenda emergente para o novo milênio. São Paulo: Unesp.

Lynn, L. E. (2012). The Many Faces of Governance: Adaptation? Transformation? Both? Neither? In: Faur-Levi, D. Oxford Handbook of Governance. New York: University Press.

Novaes, H. T., Dias, R. (2009). Contribuições ao Marco Analítico-Conceitual da Tecnologia Social. In: Dagnino, R. (Org.). Tecnologia social: ferramenta para construir outra sociedade. Campinas: IG/UNICAMP. 
Alexandre Castilhos, et AL. GovernanÇa APlicada Às tecnologias Sociais para populaÇões em SituaÇão...

Ostrom, E. (1990). Governing the Commons: The Evolution on Institutions of Collective Action. Cambridge: Cambridge University Press.

Peters, B. G. (2012). Governance as political theory. In: Faur-Levi, D. Oxford Handbook of Governance. New York: University Press.

Rhodes, R.A.W. (2012). Waves of Governance. In: Faur-Levi, D. Oxford Handbook of Governance. New York: University Press.

Rodrigues, I., Barbieri, J. C. (2008). A emergência da tecnologia social: revisitando o movimento da tecnologia apropriada como estratégia de desenvolvimento sustentável. RAP, 42(6), 1069-94.

Santos, M. M., Coelho, G. M., Santos, D. M. (2005). Foresight, engajamento social e novos modelos de governança. In: Ciência, Tecnologia e Sociedade: Novos Modelos de Governança (pp. 281-303). Brasília: CGEE - Centro de Gestão e Estudos Estratégicos.

Schneider, V. (2012). Governance and Complexity. In: Faur-Levi, D. Oxford Handbook of Governance. New York: University Press.

Smith, A., Stirling, A., Berkhout, F. (2005). The governance of sustainable socio-technical transitions. Research Policy (Vol.34, pp. 1491-1510).

Valadão, J. A. D., Andrade, J. A., Cordeiro, J. R. (2014). Abordagens sociotécnicas e os estudos em Tecnologia Social. Revista Pretexto, 15(1), 44-61.

\section{BY-NC-ND}

